\title{
Study of Interference Wavelength Characteristics of Fiber Bragg Grating Modulated All-Fiber Inline Mach-Zehnder Interferometer
}

\author{
Lijun Li, ${ }^{1,2,3^{*}}$ Qian Ma, ${ }^{3}$ Yan Zhang, ${ }^{2}$ Maoyong Cao, ${ }^{3}$ Guina Zhang, ${ }^{2}$ Lu Jiang, ${ }^{2}$ \\ Chunting Gao, ${ }^{2}$ Jia Yao, ${ }^{2}$ Yidan Li, ${ }^{2}$ Shunshun Gong, ${ }^{2}$ and Wenxian $\mathrm{Li}^{2}$ \\ ${ }^{1}$ State Key Laboratory of Mining Disaster Prevention and Control Co-founded by \\ Shandong Province and the Ministry of Science and Technology, Shandong University of Science and Technology, \\ Qingdao, 266590, China \\ ${ }^{2}$ College of Electronics, Communication and Physics, Shandong University of Science and Technology, \\ Qingdao, 266590, China \\ ${ }^{3}$ College of Electrical Engineering and Automation, Shandong University of Science and Technology, \\ Qingdao, 266590, China
}

(Received March 22, 2016; accepted June 20, 2016)

Keywords: optical fiber sensor, Mach-Zehnder interferometer, fiber Bragg grating, all-fiber, modulation

An inline Mach-Zehnder interferometer (MZI) followed by a fiber Bragg grating (FBG) structure and its novel characteristics of interference spectrum modulated by the Bragg wavelength shift were experimentally demonstrated. By changing the Bragg wavelength of the FBG, reflection cladding modes and core mode modulation was realized. In this structure, the modulation was expressed by the MIZ interference spectrum changes with the Bragg wavelength shift. Different interference intensity dip wavelengths coming from the interference of different cladding modes and core mode of the MZI were observed. A higher-order dip wavelength showed a clearly drift to the same direction with the FBG wavelength and the lower order interference dips had no obvious change. The sensitivity of this higher order dip wavelength change to the Bragg wavelength is 3.78. This dip wavelength to the FBG surrounding temperature was $41.5 \mathrm{pm} /{ }^{\circ} \mathrm{C}$, which was much higher than that of the $\mathrm{FBG}\left(10.8 \mathrm{pm} /{ }^{\circ} \mathrm{C}\right.$ in our experiments $)$.

\section{Introduction}

Optical fiber sensors based on an all-fiber inline Mach-Zehnder interferometer can be used for optical communications and sensing technology, such as all-fiber filters, attenuators and sensors of temperature, refractive index, pressure, strain, liquid level, and curvature. ${ }^{(1-3)}$ Recently, an optical fiber Mach-Zehnder interferometer (MZI) cascaded or inscribed on an optical fiber Bragg grating (FBG) has attracted significant interest because of its special spectrum characteristics and refractive index sensing. New kinds of refractometers have been proposed based on a single modemultimode-single mode (SMS) fiber structure followed by a FBG or chirp FBG. The FBGs were imprinted on a standard single-mode optical fiber (SMF) and were very closely $(5 \mathrm{~mm})$ spliced with a multimode fiber. In these experiments, the FBG reflected the core mode to the cladding of the multimode optical fiber (MMF), and two reflection cladding Bragg wavelengths were observed.

"Corresponding author: e-mail: nankaillj123@163.com

http://dx.doi.org/10.18494/SAM.2017.1346 
The relationship between the wavelength and the effective refractive index (RI) can be expressed as: $\lambda=2 n_{\text {eff }}(n) \wedge$, where $\wedge$ is the Bragg grating period, $n_{\text {eff }}$ is the $n$th mode effective RI of the cladding and core modes. The effective RI of the cladding modes will change with the surrounding RI but the core mode remains unchanged. So by measuring the relative Bragg wavelength shift between the core and the cladding Bragg wavelengths, the surrounding RI could be determined. . $^{(4,5)}$ Yue Ma et al. proposed a reflective refractometer based on a thin core (TCF) sandwiched between two single mode fibers followed by FBG. The reflection spectrum from this structure occurs at two well-defined wavelength bands, corresponding to the Bragg core mode and cladding modes. The peak power of the core mode reflection shifts linearly with the surrounding RI of the MMF.(6) Gu et al. experimentally demonstrated a reflective refractometer with high RI sensitivity based on a tilted FBG inscribed on a high photosensitive TCF by detecting changes in the cladding modes. ${ }^{(7)}$ From above research, ${ }^{(4-8)}$ it can be found that in addition to a core mode, cladding modes can also be coupled to the MZI by following it with an FBG, which will cause the MIZ spectrum to be modulated by the FBG. The characteristic of the FBG modulated MZI spectrum relate to the core mode, numerous cladding modes and reflection Bragg modes, among others. If the reflection wavelength of the FBG is changed, the spectrum of MZI would be modulated by this change. This property of MZI followed with the FBG can be used to develop a variety of sensors, filters, and optical switches.

In this study, the characteristics of an interference spectrum modulated by the Bragg wavelength of an inline MZI following a FBG of an all-optical-fiber structure were experimentally demonstrated. The modes modulation of the MZI was realized by changing the Bragg wavelength of the FBG. The interference spectrum coming from the interference between different order cladding modes and core mode modulation performance was observed. A higher-order dip wavelength showed a clear drift in the same direction as that with the FBG, with 3.78 times higher sensitivity than that of the FBG.

\section{Sensor Structure and Principle}

Figure 1 shows a schematic diagram of a MZI cascaded with a FBG. The structure consists of a section of uncoated TCF spliced between two short sections of MMF. The TCF is $15 \mathrm{~mm}$ long with a 5/125 $\mu \mathrm{m}$ core/cladding diameter ratio. Two sections of MMFs are spliced with the two ends of the TCF. Each is $5 \mathrm{~mm}$ long with a $105 / 125 \mu \mathrm{m}$ core/cladding diameter ratio and a 0.22 numerical aperture (NA), Nufern pure silica core specialty multimode optical fiber. Two sections of Corning standard SMFs, which have an $8.2 / 125 \mu \mathrm{m}$ core/cladding diameter and a 0.14 NA spliced at the two ends of the optical fiber structure of the MTM, function as input and output optical fiber, respectively. The FBG is imprinted on a standard SMF, which has a grating length of $10 \mathrm{~mm}$.

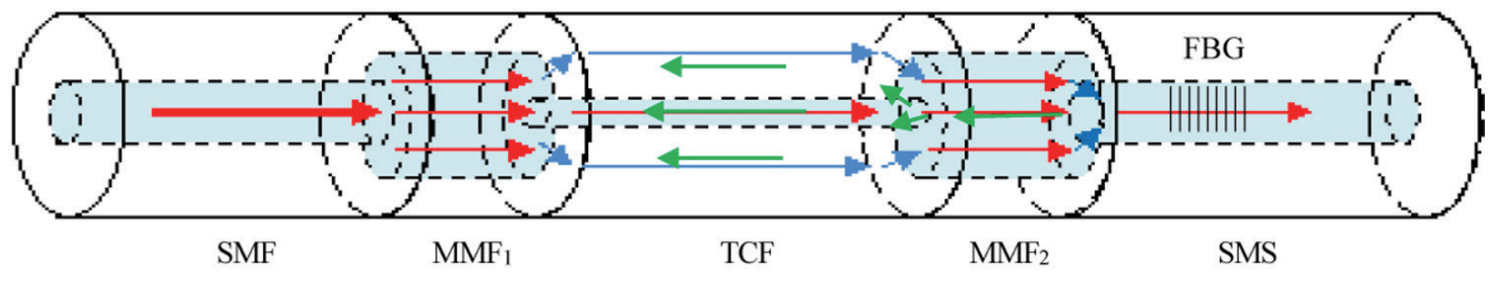

Fig. 1. (Color online) Schematic diagram of a MZI cascaded with a FBG. 
When the light is injected into the MZI from the input SMF, multiple modes will be excited and will propagate within $\mathrm{MMF}_{1}$. Assuming the field profile within the $\mathrm{MMF}_{1}$ as $\varphi_{m}(r)$, the input field at the $\mathrm{MMF}_{1}$ can be written as ${ }^{(6)}$

$$
E(r, 0)=\sum_{m=1}^{M} b_{m} \varphi_{m}(r),
$$

where $E(r, 0)$ is the core eigenmode of the input $\mathrm{SMF}, \varphi_{m}(r)$ is the $m$ th eigenmode of the $\mathrm{MMF}_{1}, b_{m}$ is the excitation coefficient for each mode, which can be expressed as

$$
b_{m}=\frac{\int_{0}^{\infty} E(r, 0) \varphi_{m}(r) r d r}{\int_{0}^{\infty} \varphi_{m}^{2}(r) r d r} .
$$

After propagation distance within the $\mathrm{MMF}_{1}$ section, the field can be expressed as

$$
E(r, z)=\sum_{m=1}^{M} b_{m} \varphi(r) \exp \left(i \beta_{m} z\right)
$$

where $\beta_{m}$ is the propagation constant of the $m$ th eigenmode within the $\mathrm{MMF}_{1}$.

For the light coupled from the $\mathrm{MMF}_{1}$ into the TCF, a portion of the light entered the TCF core while the remaining portion is coupled into the TCF cladding. Similarly, at the TCF and $\mathrm{MMF}_{2}$ splice point, partly cladding modes of the TCF again coupled into the $\mathrm{MMF}_{2}$, and multiple modes of $\mathrm{MMF}_{2}$ consequently coupled into the lead-out SMF. The coupling coefficients can be expressed as follows

$$
c_{n}=\frac{\int_{0}^{\infty} E^{\prime}(r, z) \phi_{n}(r) r d r}{\int_{0}^{\infty} \phi_{n}^{2}(r) r d r}
$$

where $E^{\prime}(r, z)$ is the field of the output of $\mathrm{MMF}_{2}, \phi_{n}(r)$ is the $n$th mode within the output SMF and $n$ $=1$ represents the core mode, and $n>1$ represent cladding modes.

When these modes arrive at the FBG, they will be reflected by the FBG and the reflection wavelengths can be expressed as follows ${ }^{(2)}$

$$
\lambda_{n}=2 n_{e f f(n)} \Lambda,
$$

where $\lambda_{n}$ is the reflection wavelength of the FBG, $n_{\text {eff }(n)}$ is the $n$th effective refractive index of the output SMF ( $n=1$ represents the core mode and $n>1$ represent cladding modes), and $\Lambda$ is the Bragg grating period.

The transmission spectrum of this MZI can be expressed as ${ }^{(2)}$

$$
I_{\text {out }}=\left[E(r, z) c_{n} \gamma\right]^{2}+\left[E(r, z)\left(1-c_{n}\right)\right]^{2}+2 E_{0}^{2} c_{n}\left(1-c_{n}\right) \gamma \cos \frac{2 \pi \cdot \Delta n_{e f f} L}{\lambda},
$$

where $E(r, z)$ is the electrical field amplitude of the field launched into the MZI, $\gamma$ is the propagation loss of cladding modes, $\Delta n_{\text {eff }}$ is the difference of the effective refractive indices between core and cladding modes, $L$ is the geometry length of the MZI, and $\lambda$ is the wavelength of the propagating 
light. The intensity dip appears at wavelengths as follows:

$$
\lambda_{\text {dip }}=\frac{2 \Delta n_{e f f} L}{2 n+1} .
$$

\section{Experimental Setup and Results}

Experiments based on the above structure were carried out. The experimental setup is shown in Fig. 2. The BBS is a broad band light source, whose wavelength ranges from $1531 \mathrm{~nm}$ to 1560 $\mathrm{nm}$. A water bath-heating box is used to change the surrounding temperature of the FBG. Two fiber holders are kept free from the influence of bend and stress. A YOKOGAWA-AQ6370 optical spectrum analyzer (OSA) with $0.05 \mathrm{~nm}$ resolution measured the spectrum. In our experiments, a water bath-heating box was used to heat only the FBG. A thermal baffle was used to deflect heat spread to the outside which would affect the interference spectrum of the MZI.

The transmission spectrum of the MZI followed with a FBG while the FBG was immersed in water at a temperature of $29.2{ }^{\circ} \mathrm{C}$ is shown in Fig. 3. The intensity dip wavelengths and intensity visibility of the interference spectrum of this sensor can be calculated in theory by Eqs. (7) and

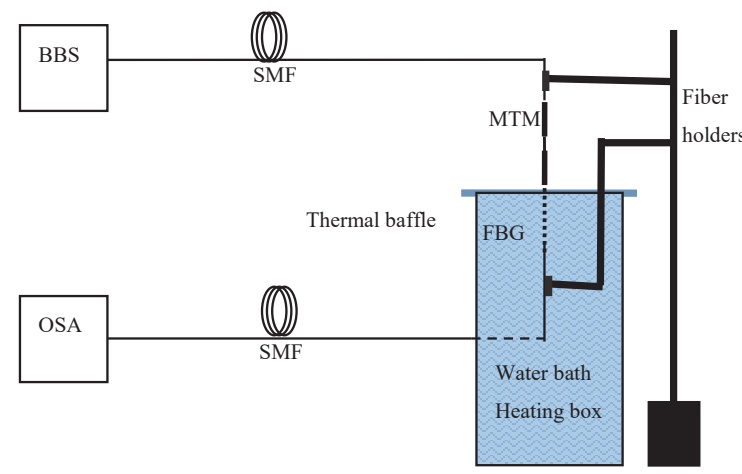

Fig. 2. (Color online) Experimental setup.

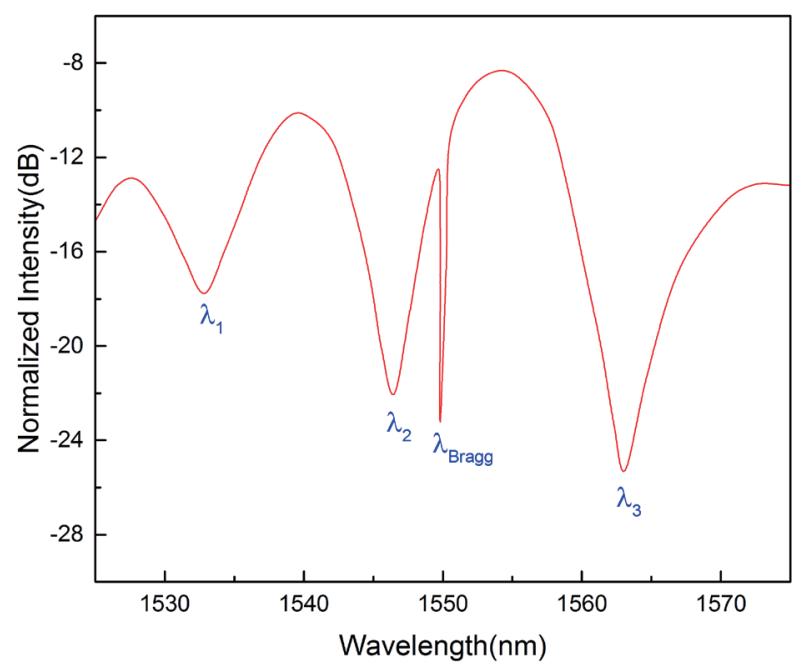

Fig. 3. (Color online) Transmission of MZI followed with the FBG in $29.2{ }^{\circ} \mathrm{C}$. 
(6). In Fig. 3 , there are three intensity dip wavelengths, at $\lambda_{1}=1532.8 \mathrm{~nm}, \lambda_{2}=1546.3 \mathrm{~nm}$, and $\lambda_{3}=$ $1563 \mathrm{~nm}$, respectively. The FBG central wavelength $\left(\lambda_{\text {Bragg }}\right)$ was $1549.96 \mathrm{~nm}$ with $90 \%$ reflectivity and $0.00 \mathrm{~nm} 3 \mathrm{~dB}$ bandwidth. The grating period in our experiments is $\Lambda$ and $2 \Lambda$ is $1073.3 \mathrm{~nm}$.

A water bath-heating box was used to change the surrounding temperature of the FBG from 29.2 to $94.1{ }^{\circ} \mathrm{C}$ to shift the Bragg wavelength. Figure 4 shows the interference spectrum of MZI modulated by the FBG as a function of its surrounding temperature. From this figure, it can be seen that the dip $\lambda_{3}$ shifted with $\lambda_{\text {Bragg }}$ and that the dip wavelength $\lambda_{1}$ and $\lambda_{2}$ remained nearly unchanged with the $\lambda_{\text {Bragg. }}$. It can also be seen that all the intensity visibility of dip wavelengths were changed with the $\lambda_{\text {Bragg }}$ change. This can be explained as follow: From Eq. (5), we can determine that the modes number $n$th changes with the reflection wavelength $\lambda_{\text {Bragg. }}$. From Eq. (4), the coupling coefficients $c_{n}$ change with the $n$ th, and from Eqs. (6) and (7), the intensity and dip wavelengths of the MZI interference spectrum changed with the $c_{n}$. So we can conclude that the intensity visibility and dip wavelengths are all changed with the shift of the $\lambda_{\text {Bragg. }}$. Longer interference intensity dip wavelengths corresponding to higher-order modes have higher sensitivity for mode changes than to shorter wavelength. In these experiments, only the longer interference wavelength of MZI could sense the modes change in the core and the cladding of the sensor.

The detailed performance of $\lambda_{1}$ and $\lambda_{2}$ modulated by $\lambda_{\text {Bragg }}$ is shown in Fig. 5(a). These two wavelengths are almost unchanged with the $\lambda_{\text {Bragg. }}$. Figure $5(\mathrm{~b})$ is the performance of $\lambda_{3}$ change with $\lambda_{\text {Bragg }}$. By calculating the slope of the points, a nearly linear modulation occurs between $\lambda_{3}$ and $\lambda_{\text {Bragg }}$ with a modulation ratio of 3.78. Figure 6(a) shows the $\lambda_{\text {Bragg }}$ changes with bath temperature, and that its sensitivity was $10.8 \mathrm{pm} /{ }^{\circ} \mathrm{C}$. Figure $6(\mathrm{~b})$ is the $\lambda_{3}$ versus the temperature of the FBG.

When light travels along the fiber, MZI couples part of the core mode to a relatively large wavelength range cladding mode. Both the cladding mode and the core mode continue to travel along the fiber until they reach the FBG. The core mode and cladding mode are reflected back at some wavelengths determined by the parameters of the FBG. This means that the core modes and cladding modes are all affected by the Bragg wavelength. Both the cladding and core mode

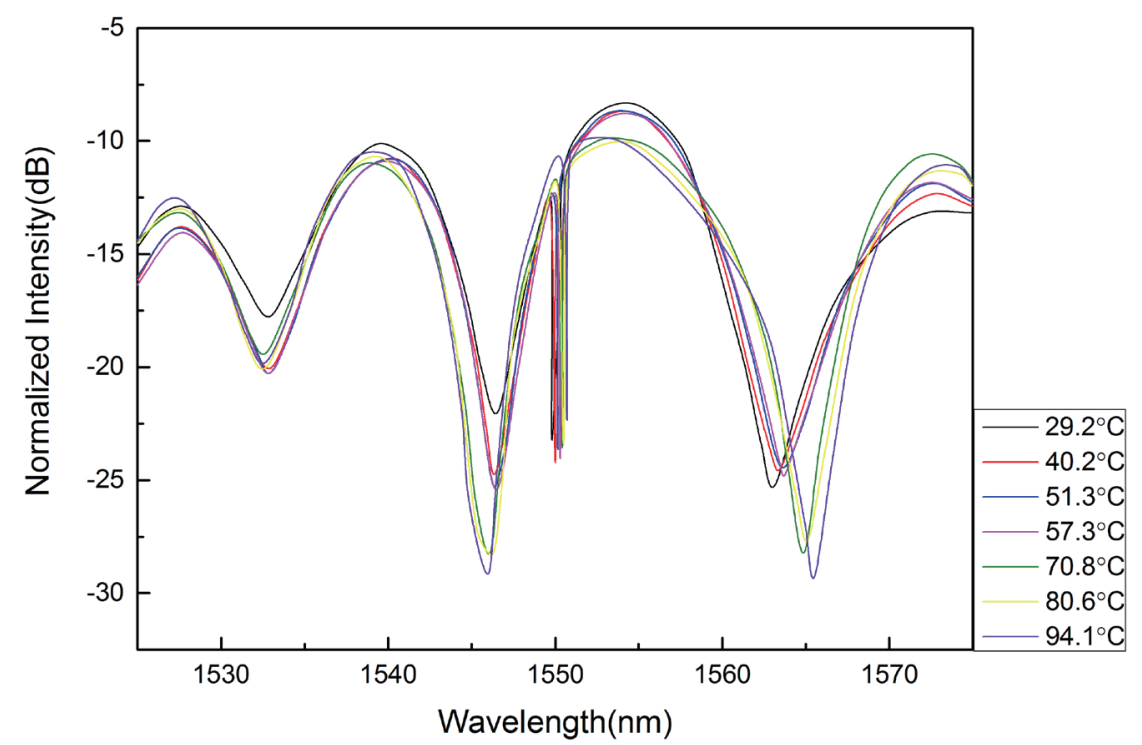

Fig. 4. (Color online) Spectral response of interference intensity dips as a function of Bragg wavelength. 


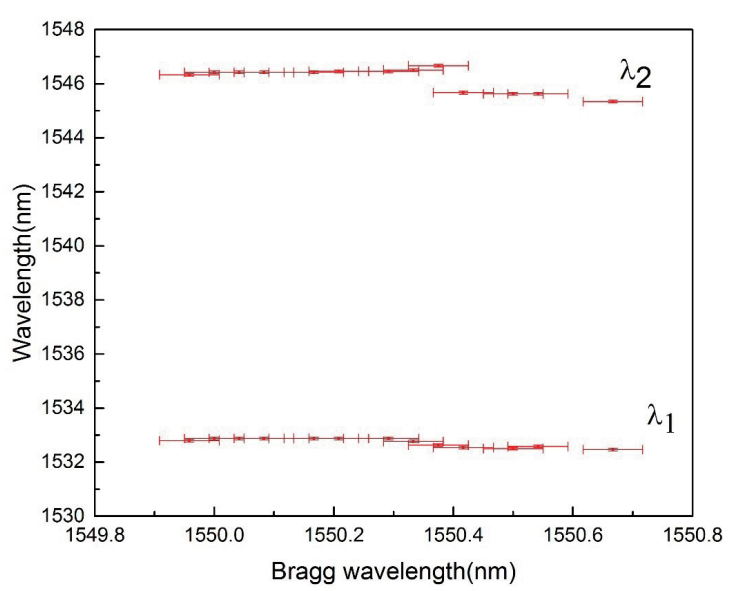

(a)

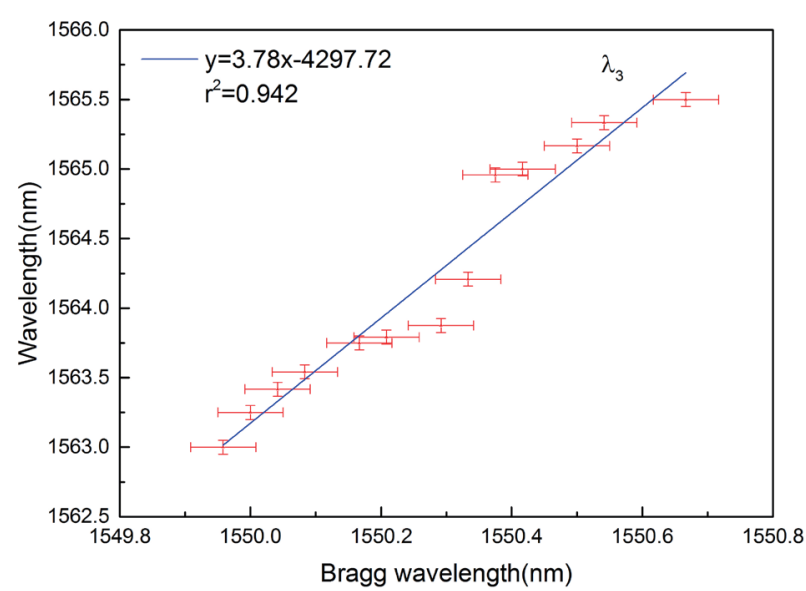

(b)

Fig. 5. (Color online) Measured MZI dip (a) $\lambda_{1}, \lambda_{2}$ and (b) $\lambda_{3}$ wavelengths versus Bragg wavelength $\lambda_{\text {Bragg }}$ and its linear fits.

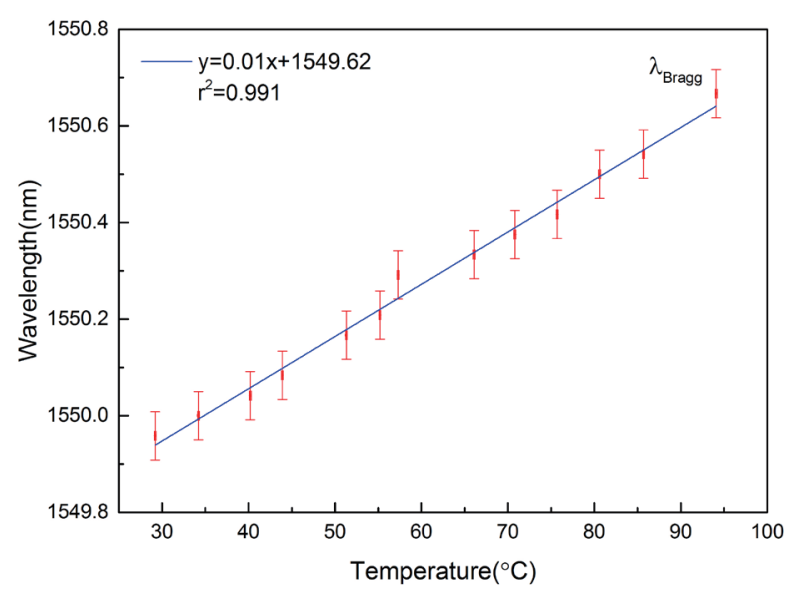

(a)

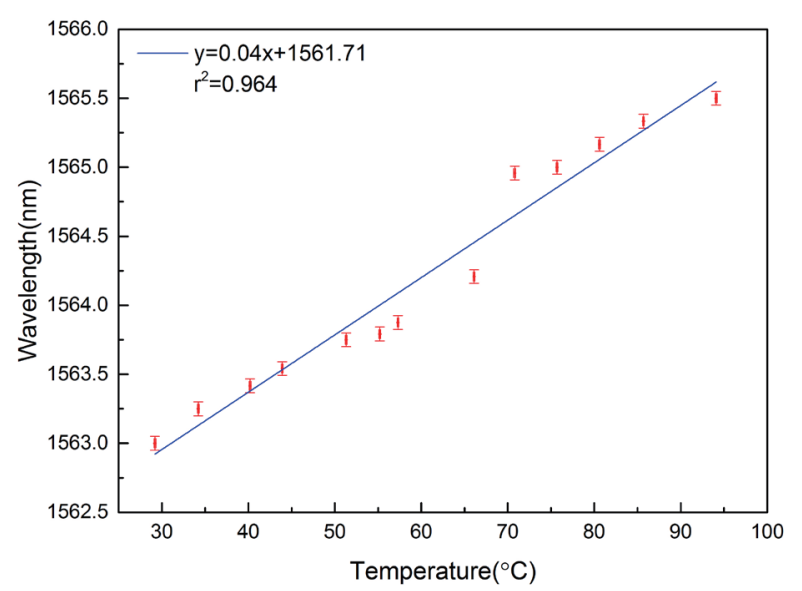

(b)

Fig. 6. (Color online) Measured (a) $\lambda_{\text {Bragg }}$ and (b) dip $\lambda_{3}$ versus the surrounding temperature of the FBG and their linear fits.

reflection bandwidths are narrow because of the narrow bandwidth of FBG. Longer interference intensity dip wavelengths corresponding to higher-order modes have higher sensitivity for mode changes than to shorter wavelength. So in these experiments only the longer interference wavelength of MZI could sense the slight change in the core and cladding modes.

\section{Conclusions}

In summary, we have fabricated an all-fiber inline MZI followed by a FBG structure by using studies of FBG modulation characteristics. Different core modes and cladding modes are modulated by changes of the FBG central wavelength. This is a slight modulation of modes because of the narrow reflection bandwidth of the FBG. In our experiment, three interference intensity dip wavelengths of MZI were observed. The enveloping MZI transmission arrives from 
interference between core modes and several cladding modes, and is modulated by fluctuations in the core modes or cladding modes. ${ }^{(1-3)}$ In our experiment, the lower order mode interference dip wavelengths were almost unchanged as the wavelength of the FBG shifted, and the higher order interference dip wavelength shifted with a FBG with a high modulation ratio (3.78). This kind of device could effectively improve the sensitivity of slight mode changes, which makes it attractive for many communication and sensing applications.

\section{Acknowledgements}

This work was supported by the Shandong Provincial Natural Science Foundation of China (Grants Nos. ZR2009AM017 and ZR2013FM019), the State key Laboratory Breeding Base for Mining Disaster Prevention and Control Open Fund Research Project (Fund No. MDPC201602), the National Postdoctoral Project of China (Grants Nos. 200902574 and 20080441150), Shandong Provincial Education Department Foundation of China (Grants No. J06P14).

\section{References}

1 L. V. Nguyen, D. Hwang, S. Moon, D. Seung Moon, and Y. Chung: Opt. Express 16 (2008) 11369.

2 L. Li, L. Xia, Z. Xie, and D. Liu: Opt. Express 20 (2012) 11109.

3 C. R. Liao, D. N. Wand, and Y. Wang: Opt. Lett. 38 (2013) 757.

4 Q. Wu, Y. Semenova, B. Yan, Y. Ma, P. Wang, C. Yu, and G. Farrell: Opt. Lett. 36 (2011) 2197.

5 A. Sun and Z. Wu: IEEE Photon. Technol. Lett. 24 (2012) 413.

6 Y. Ma, X. Qiao, T. Guo, R. Wang, J. Zhang, Y. Weng, Q. Rong, M. Hu, and Z. Feng: Opt. Lett. 37 (2012) 323.

7 B. Gu, W. Qi, J. Zheng, Y. Zhou, P. P. Shum, and F. Luan: Opt. Lett. 39 (2014) 22.

8 M. D. Baiad, M. Gagné, S. L. Renaud, E. D. Montigny, W. J. Madore, N. Godbout, C. Boudoux, and R. Kashyap: Opt. Express 21 (2013) 6873. 